\title{
Algunos modos de historiar la educación especial en Colombia: UNA MIRADA CRÍTICA DESDE LA HISTORIA DE LA PRÁCTICA PEDAGÓGICA ${ }^{1}$ M ODES OF MAKING SPECIAL EDUCATION HISTORY IN COLOMBIA: A CRITICAL VIEW ABOUT THE HISTORY OF PEDAGOGIC PRACTICES
}

Alexander Yarza DeLOSRÍOS²

RESU M EN : teniendo en cuenta el desprecio aparentehacia la historia en al gunas investigaciones y en la mayoría de la formación de educadores especiales, se presenta un anál isis sobre el lugar de la historia de la educación especial en el conjunto de historias de la educación y la pedagogía, y un estado del arte sobre los diferentes modos de historiar la educación especial en Colombia. Para finalizar, se propone un análisis crítico desdela historia dela práctica pedagógica posi bi litando pensar los aportes de las distintos modos dehistoriar a futuras formas de investigación histórica que contribuyan a ponerle un alto al incremento y expansión de la desmemorización de la cultura, la historia, la pedagogía y, para nuestro caso, de la educación especial.

PA LA BRA S CLA VES: historia de la educación especial en Colombia; historia de la práctica pedagógica, educación especial.

A BSTRACT: keeping in mind theapparent scorn toward thehistory in someinvestigations and in most special educator's formation, an analysis is presented on the place of the history of the special education in the group of education and pedagogy's histories, and a state of the art on the different ways to make history the special education in Colombie. To conclude, it intends a critical analysis from the history of the pedagogic practice facilitating to think the contributions from the different ways to make history to future forms of historical investigation that contribute to put a high one to theincrement and expansion of the give-memorization of the culture, the history, the pedagogy and, for our case, of the special education.

KEYWO RD S: history of the special education, History of the pedagogic practice; special education.

La historia del saber vivifica las comunidades disciplinares, es parte de las certezas que deben cohesionarlas. El desprecio por la historia es hijo de una estéril actitud que puederesumirse en la frase: "tierra de nadie".

Olga Lucía Zuluaga y Jesús Alberto Echeverri

\footnotetext{
${ }^{1}$ Este artículo es un producto de la investigación: “El Rastro de la Diferencia. Historia dela Práctica Pedagógica de la Educación Especial en Colombia, 1845-1886", financiada por el Comité para el Desarrollo de la Investigación, Universidad de Antioquia, 2002-2004. A simismo, es una versión ampliada de una ponencia presentada en el XIII Congreso Colombiano deH istoria, Bucaramanga, 22 a 25 deA gosto de2006. A Wayra, Fruto del amor entrela tierra einti

${ }^{2}$ Licenciado en educación especial, Profesor de cátedra, Facultad deEducación, Universidad de A ntioquia. Investigador asociado del Grupo H istoria de la Práctica Pedagógica en Colombia. Investigador fundador del Grupo Educación Especial, Inclusión y D iversidad. Facultad de Educación, Universidad deAntioquia. yacosume@yahoo.es
} 
[...] Cuando depronto todo ha concluido y sól o nos queda un bloquede piedra con unas inscripciones, siempre que al guien se haya acordado o haya tenido el tiempo necesario para hacer grabar esas letras en el mármol. Pasa el tiempo, Ilueve y brilla el sol y llega un día en que nadie recuerda el nombre y lo que dicen esas letras, nada importa ya. Quizás por eso, si uno puede dirigirse a al guno, cuanto más extraño mejor, y darleal go, lo que sea: un pliego de papel o cualquier otra cosa que nada signifique por sí misma, aunque ellos no lo lean ni lo guarden, ni se preocupen siquiera por destruirlo o arrojarlo, ya es al go porque ha sucedido y puede ser recordado, pasando de una mano a otra, de una inteligencia a otra, al menos es como un grabado, al go que deja rastro, algo que existió un día, pues de otro modo no podría morir también; en tanto queel bloque demármol jamás podría ser presente, puesto quetampoco llegará a ser pasado, es incapaz de morir o terminar.

WilliamFaulkner

\section{INTRODUCCIÓN}

Las siempresabias palabras dela profesora Olga Lucía Zuluaga y demi maestrojesúsA lberto Echeverri, marcan el sentido más profundo deesteescrito. El desprecio sueleser la puerta de entrada para un olvido contundente. Si bien nuestras comunidades académi cas de historiadores dela educación y la pedagogía han intentado consolidar un público quevalore y resignifique esas historias (mediantecongresos, forosy seminarios, grupos, centros y redes deinvestigación, publicaciones seriadas, cursos y tesisen el pregrado o posgrado, maestrías y doctorados), no puedeolvidarse el hecho contundente de que la mayoría de maestros en formación y maestros de escuela (por no decir algunos investigadores de las didácticas, el currículo y la educación), todavía sienten una especie de desprecio febril y automático por la historia, por su historia, es decir, por sí mismos en tanto sujetos históricamente fechados y situados (como nos enseñó a decir Paulo Freire).

Si estefenómeno del "desprecio" funciona tanto para la historia dela educación como para la historia social, política, económica y cultural del país, imagínense el estado en que se encuentra el olvido de las historias de la educación especial en Colombia. Además, como plantea Fernando Bárcena (2001, p. 69): "Vivimos en sociedades modernas ancladas, en buena medida, en un cultura del olvido, casi amnésicas [...] El olvido dela autoridad de pasado y dela tradición es un olvido deliberado y, en parte, para la cultura de la modernidad, un olvido sanador del espíritu ilustrado. Estos rasgos de nuestra modernidad afectan profundamente a la educación." En térmi nos generales, se puede afirmar, no sin cierta precaución, queen la mayoría de los programas de pregrado en educación especial de Colombia (FACULTAD DE EDUCACIÓN, 2006), es decir, en Ios procesos deformación inicial de "educadores especiales", los problemas históricoepistémicos y pedagógicos de la constitución desu "saber", "campo disciplinar" o "conocimiento profesional", han sido y están siendo omitidos u olvidados, relegados a un papel secundario, ínfimo, periférico, excéntrico y hasta “inútil”. 
Las historias de la educación especial pueden ser consideradas como un fragmento minúsculo en un bloque de piedras o de mármol de la cultura en nuestra sociedad. El olvido y el desprecio no pueden perpetuar las formas de relación con el presente, con esas prácticas político-pedagógicas detransformación local de las condiciones reales de existencia en la educación especial y en los procesos de integración educativa y social. Nuestro presente no puede ponerle una espal da insulsa a la historia. Es necesario eindispensable escribir al go en unas hojas de papel, aunque, como bien dice William Faulkner (1951), "no lo lean ni lo guarden, ni sepreocupen siquiera por destruirlo o arrojarlo", en el sentido que "ya es al go porque ha sucedido y puedeser recordado [...] al menos es como un grabado, algo que deja rastro, algo que existió un día, pues de otro modo no podría morir también." La muerte no perdona el desprecio estéril de un olvido despiadado; además, no podemos dejar de recordar que a veces solemos y podemos ser unos navegantes que buscan un territorio, una tierra quenos pertenecey queen verdad estierra dealguien.

\section{La educación especial en algunas historias de la educación y la pedagogía}

En Colombia existen un conjunto de “historiasclásicas" dela educación y la pedagogía: La evolución educativa en Colombia de Luis Antonio Bohórquez Casallas (1956), Educación e ideología en Colombia delvon LeBot (1979), El proceso de la educación en la república (1830-1886) y La educación durante los gobiernos liberales 1930-1946 de Jaime Jaramillo Uribe (1984, 1989), La educación en Colombia. 1880 1930 deRenán Silva Olarte(1989), La educación en Colombia: 1918-1957 y La educación en Colombia: 1958-1980 deAlineHelg (1987) desdela Nueva Historia deColombia. Pero también han aparecido unas más recientes de Javier Sáenz, Oscar Saldarriaga y A rmando Ospina (1997), Antonio Cacua Prada (1997), Martha Herrera (1999), Zandra Pedraza (1999), Humberto Quiceno (2003), Oscar Saldarriaga (2003), entre otras. Todas éstas y muchas investigaciones y ensayos más han permitido hablar deun campo deestudios históricos en educación y pedagogía para nuestro país. ${ }^{3}$

En este sentido, Jorge Orlando Castro (2000) realizó un estado de la cuestión sobre las investigaciones históricas realizadas en educación y pedagogía durante 1990 y 1999. En su estudio nos habla dehistorias globales, historias sociales ehistoria dela práctica pedagógica; pero, además, es necesario recordar quetambién sehan realizado historias delas ideas pedagógicas ehistorias económicas y políticas (MARTÍNEZBOOM;ZULUAGA apud MARTIINEZ; NARODOWSKI, 1996) einduso historias dela pedagogía como historia de la cultura (JARAMILLO URIBE, 1978).

\footnotetext{
${ }^{3}$ Esta situación puede confirmarse en el Panel 11 del X III Congreso Colombiano de H istoria realizado en agosto de2006, el cual planteó al gunos debates en torno a la investigación en la H istoria de la Educación en Colombia, específicamente con trabajos sobre historiografía de la educación en la N ueva Granada, de estudios sobre la infancia, sobrela modernización educativa en Santander a finales del siglo XIX, sobre el desajuste del aparato escolar y la crisis del desarrollo expansivo en el Sistema Educativo a finales del siglo XX, sobrehistorias dela educación especial, sobreel programa educativo para la paz y la reconciliación nacional y sobrela educación pública en Bogotá durantela década de 1930. (MEMORIASXIII Congreso Colombiano de H istoria, 2006)
} 
Son pocas las historias de la educación y la pedagogía en Colombia que han hablado directamente sobre la historia de la educación especial. ${ }^{4}$ Los "estudios históricos tradicional es" o historias gl obales la retoman en tanto datos periféricos o como anécdotas hiladas en el progreso general y lineal dela educación en el país. La mayoría de las “historias sociales" no la mencionan. Sin embargo, una historia dela práctica pedagógica en Colombia entre1903 y 1946la hizo visible en tanto uno de los espacios de apropiación delapedagogía activa experimental. Al decir de Javier Sáenz, Oscar Saldarriaga y Armando Ospina, la “educación de anormales" se entiendecomo el segundo espacio de apropiación dela Pedagogía Activa en Colombia5:

\begin{abstract}
A partir del saber médico, en los años veinteseintroducirían al gunas prácticas del examen escolar y dela Pedagogía activa en escuel as para anormales y para menores delincuentes -tanto privadas como públicas-, principal mente en el departamento deA ntioquia, las cuales transformaron la enseñanzay el régimen carcelario de estos establecimientos. A partir de su reorientación, estas instituciones seconvirtieron en escuelas examinadoras, regidas por los principios y las prácticas de la vertiente experimental europea de la Pedagogía activa. Tales instituciones dirigieron su mirada hacia la detección y corrección de anormalidades psíquicas y físicas y desarrollaron prácticas de clasificación escolar y orientación profesional (1997, v. 2, p. 49)
\end{abstract}

Esta mirada pedagógica hace visible un innombrado que mereció un análisis minucioso, cercano y sistemático sobre sus formas específicas de apropiación einstitucional ización en Colombia. La pregunta por la pedagogía activa implicó acercarse a la educación especial para percibir de otro modo los relatos históricos sobre la educación colombiana a principios del siglo XX.

\title{
Historias de la educación especial en Colombia
}

En nuestro país, las contadas historias dela educación especial pueden clasificarse de la siguiente manera: "historia de datos", "historia institucional" e "historia social". Cada una de estas historias tiene referentes metodológicos y conceptuales distintos, los cuales hacen quela historia que cuentan sea más o menos diferente, aunque con varios puntos en común. Se puede decir que la principal diferencia entre cada modo de hacer historia radicaría en la forma de entender y trabajar el archivo y deorganizar, analizar einterpretar la información, los datoso los registros. Trabajo de archivo e interpretación que varía bien sea desde una

\footnotetext{
${ }^{4}$ En este punto es ineludible resaltar que la historia de la educación especial también ha sido abordada tangencialmentedentro de una historia de la psiquiatría en Colombia (ROSELLI, 1968), mostrando la necesidad y posibilidad dearticular los modos deanalítica histórica pedagógica desde unos referentes conceptuales que permitan escudriñar las filiaciones y procedencias discursivas que han servido de sustento a la práctica y los discursos en la educación especial (Cfr. YARZA , 2006a, 2006b, YARZA y RODRÍGUEZ, 2005)

${ }^{5}$ El primer espacio de apropiación son algunos escritos deintelectual es modernos, los cuales estaban dispersos en revistas y tesis; el segundo son las facultades de medicina y las prácticas del examen escolar; un tercer espacio fue el Gimnasio Moderno, el cuarto fue el uso deal gunas nociones provenientes de las teorías de la evolución, la criminología, la psiquiatría y la medicina, el discurso de la sociobiología especulativa y las discusiones sobrela degeneración de la raza (SA ENZ, SA LDARRIAGA Y OSPINA, 1997)
} 
mirada general sobre datos, fechas y sujetos, desdelas transformaciones internas de al gunas instituciones de "educación especial" o desde las relaciones entre las instituciones y los discursos sociales, económicos y políticos. También es necesario mencionar quequienes practican estos "modos dehistoriar" no han sido solamente "historiadores", sino también administradores de la educación y maestras o maestros interesados en la problemática.

La historia de datos enfatiza en una mirada cronológica de los hechos tomando como referente principal un imaginario sobreel pasado construido desde Ios "descubrimientos" de la actualidad. Los trabajos agrupados desde esta perspectiva plantean distintos períodos para la historia de la educación especial, contemplando similitudes enormes entre sí: similitud en los acontecimientos, en la extensión de los períodos o en la forma de análisis de los datos.

Joséjaime Díaz Osorio (1987) plantea quela educación especial en el país comienza desde inicios del siglo XX. Este inicio se encuentra marcado por disposiciones legales aisladas y por acciones filantrópicas de algunas personas. Sitúa los antecedentes de la "educación especial" en A ntioquia hacia finales del siglo XVIII y mediados del XIX, pero sostiene que "[...] la educación especial, propiamentetal, arranca con la Escuela de Trabajo San Joséy la Escuela deCiegos y Sordos." (DÍAZ OSORIO, 1987, p. 18); la primera fundada en 1914 por Ordenanza 5 y la segunda por Decreto 4 de 1925. Luego deestablecer una somera descripción sobre estas instituciones (legislación, trascripción de reglamento, vicisitudes económicas, traslado de Dirección, entre otros aspectos), Díaz Osorio efectúa un recuento histórico lineal y progresivo sobre la legislación referida a la educación especial desde 1925 hasta la década de 1970. Esta narrativa histórica rescata las Leyes o Decretos y lo que disponeen el ámbito nacional o regional: establecimientos de instituciones, adquisición de terrenos, creación de Fondos, Federaciones e Institutos, sobresueldos para los profesores. Díaz Osorio divideen cinco momentos el breverecuento histórico dela educación especial, a saber: 1. El limitado a través de la historia; 2 . Inicios de la educación especial en el país; 3. A ntecedentes de la Educación Especial en el departamento deAntioquia; 4. Nacimiento dela Educación Especial en Antioquia; y 5. A vances de la Educación Especial en el País y en el departamento.

Lucy Salinas (1988), Directora dela División deeducación Preescolar y Educación Especial del Ministerio deEducación Nacional en 1987-1988, propone tres períodos: período inicial definales del siglo XIX-1960, segundo período entre 1960-1970, y el tercero seinicia en 1970-1988. El primer período secaracteriza por “[...] la aparición de las primeras escuelas dedicadas a la atención de niños con limitaciones visuales y auditivas, el establecimiento de estímulos a los docentes que laboran en esta área y la creación de impuestos con destino a institutos de educación especial". (SA LINAS, 1988, p. 47) A segura quela formación institucional dedocentes serealizó al final dela década de1950 en el instituto deespecialización del magisterio del Distrito Especial deBogotá, en el Instituto Nacional para Ciegos, y en la Federación de Ciegos y Sordomudos de Colombia. “[... la tendencia de 
este período era la de ofrecer servicios segregados para los limitados visuales y auditivos, con un carácter más detipo médico asistencial queeducativo y laboral". (lbíd.)

En el segundo período, entre 1960-1970, aparecen los primeros centros deatención para niños con parálisis cerebral, asimismo “[...] secomienza a prestar los primeros servicios de capacitación laboral para adultos ciegos en el Centro de Rehabilitación para el Adulto Ciego, CRAC y para limitados neuro-musculares y ortopédicos en el Centro N acional de Rehabilitación". (SA LINAS, 1988, p. 47-48). En 1968secrea la División deEducación Especial correspondiéndole “[...] promover programas y servicios deeducación especial, tanto para alumnos subnormales como para estudiantes sobresalientes". Lo relevante de este período se reduce, según Salinas, en: 1. La aparición de la Unidad Central de Educación Especial en el Ministerio deEducación Nacional y dealgunas unidades regionales; 2. La creación del Instituto Colombiano de Bienestar Familiar encargado de atender las necesidades de las familias problemáticas e inestables; 3. La creación del Consejo Nacional de Rehabilitación; 4. La proliferación de centros de educación especial que atendían mayoritariamente a los "retardados mentales"; 5. La iniciación de formación del personal paramédico y de maestros para limitados visuales y retardados mentales en las Universidades Nacional, del Rosario y Pedagógica Nacional; y, 6. Preparación deprofesionales en el campo dela educación especial en el exterior a través de becas y préstamos en el ICETEX, Instituto Colombiano de Crédito Educativo y Estudios Técnicos en el Exterior (SALINAS, 1988, p. 48)

En el tercer período, entre 1970-1988, se crean establecimientos educativos para atender "alumnos sobresalientes": Ia Fundación Humboldt en Bogotá y el Instituto Experimental del Atlántico "José Celestino Mutis" en Barranquilla. El Instituto $\mathrm{N}$ acional para Ciegos $(\mathrm{INCI})$ y el Instituto $\mathrm{N}$ acional para Sordos (INSOR) se transforman en establecimientos públicos del sector educativo y no del sector salud. En 1974 la División deEducación Especial crea el Programa deAulas Especiales en la Escuela Regular, “[...] con esteprograma el Ministerio de Educación atiendea los niños con dificultades en el aprendizaje, especial menteen el primer grado de educación básica y a los niños con retardo mental educable." (SALINAS, 1988, p. 49). En 1976 seincluyela educación especial dentro del sistema educativo del país. En 1981 se crea el Sistema Nacional de Rehabilitación que coordina e integra los sectores salud, trabajo, justicia y educación. Lucy Salinas concluyeque "En términos generales [... ] la educación especial, en el país, ha sido vista como una acción importante dentro del sistema educativo nacional" (I bíd.)

Jorge Enrique Duarte (1989), profesor de la Universidad Pedagógica Nacional, hace referencia al trabajo construido por Lucy Salinas, donde registra tres grandes épocas o momentos históricos en la educación Especial: 1. Del nacimiento, 2. Dela niñez y 3. Dela juventud. El primero del Nacimiento se ubica entre 1950 a 1960, con la creación del Instituto N acional para Ciegos y la Federación de Ciegos y Sordomudos de Colombia con la vigilancia del Ministerio de Salud Pública. El segundo de la Niñez se ubica entre 1960 y 1970, con la fundación y 
creación de centros de rehabilitación y la apertura de programas a nivel tecnológico (el Instituto dePedagogía Infantil INPI - Bogotá) y nivel deformación universitaria (Universidad Nacional y del Rosario-Bogotá, terapias física, ocupacional y del lenguaje), seleasignan actividades y funciones específicas en el área deeducación especial al ministerio de Educación N acional. La tercera dela Juventud, se ubica entre 1971 hasta finales de 1980 (fecha depublicación del artículo), y secaracteriza por la apertura en 1974 del programaaulas especial es para niños con retardo mental y aulas remediales para niños con dificultades en el aprendizaje, con la dirección del Ministerio deEducación Nacional. (DUARTE, 1989, p. 32)

El profesor Duarte combina una visión cronológica lineal con una metáfora biológico-evolutivay, además, se distancia delas demás posturas en tanto plantea queel "nacimiento" dela educación especial se gesta solamente desdela década de 1950 (cuando hemos observado quela mayoría de estas posturas indican queemergea principios del XX: 1910-1920).

Mercy Paez Escobar (1994), profesional especializado de la División de Educación de Poblaciones Especiales del Ministerio de Educación Nacional, plantea quetanto en Colombia como en Europa y A mérica el proceso evolutivo de la educación especial ha pasado por cuatro etapas: exterminación, abandono, aceptación eintegración. Desde una mirada aparentementedistinta, Paez propone entender esta "evolución” en dos momentos o períodos: del enfoque clínico y del enfoque pedagógico.

El primero se extiende desde 1903 hasta 1974 y se fundamenta en la forma como se percibe al sujeto con deficiencia, como un ser con difíciles posibilidades de modificación por cuanto las causas de su deficiencia son "puramente orgánicas". Surgen entonces las categorías, las clasificaciones y, por consiguiente, la necesidad de agruparlos según sus características. Proliferan las instituciones porque es allí donde este tipo de personas pueden aprender, “[...] aparecen currículos especiales paral elos al currículo de la escuela regular y el acento se pone en la atención terapéutica porque el problema es más de tipo patológico queeducativo". (PAEZ, 1994, p. 9) En esteperíodo prima “[...]la institucionalización como hecho fundamental y prácticamentela única alternativa." (Ibíd.), incluyendo la creación de una gran cantidad de instituciones especiales centralizadas en diferentes poblaciones: ciegos, sordomudos, retardados, etc.

En el segundo momento, desde 1974 hasta 1994, se otorga mayor relevancia a los procesos de aprendizaje y a las dificultades que encuentran los alumnos para su progreso en la educación y la enseñanza. Esteenfoque rescata la importancia dela pedagogía sin desconocer el "impacto dela limitación", centrando su atención en "[...] los problemas de aprendizajey los recursos educativos que se requieren para satisfacer las necesi dades del alumno. El énfasis se acentúa en la escuela, y en la respuesta que ésta le de [...]" (PAEZ, 1994, p. 10). Según Paez, en este momento surgen dos conceptos: el de necesidades educativas especiales y el deeducación especial y su quehacer, insertada dentro del sistema educativo general. 
La historia institucional centra su mirada sobre los procesos históricos de las instituciones. Hugo de Jesús Pérez señala que son aquellos estudios que

[...] haceénfasis en la historia interna dela institución, enmarcada en el contexto social, económico y cultural desu surgimiento y posterior desarrollo. Interesa prestar atención a la intencionalidad del grupo de fundadores al crear la organización, analizar el tipo de demandas que se pretendían cubrir y la finalidad desu particular forma de constitución, así como los cambios que en este sentido se dieron en las últimas décadas. (PÉREZ, 2003, p. 7)

Sus principales representantes serían el trabajo pionero del profesor A ntonio Marín dela Fundación Universitaria Luis A migó (1992) sobrela historia dela Casa de Menores y Escuela deTrabajo San José (desde 1914 hasta 1990) y del historiador Hugo de Jesús Pérez (2003) sobre la Institución Educativa Francisco Luis Hernández para ciegos y sordos (1925-2000); ambas instituciones se pertenecientes al departamento deA Antioquia.

Estas historias tienen en cuenta el contexto local, regional y nacional en el que se funda la institución, las transformaciones de cada uno de los reglamentos (mostrando las permanencias y cambios en artículos, reglas, ideales, etc.), las vicisitudes de las Juntas de Dirección y sus relaciones con entidades gubernamentales (municipales o departamentales), las relaciones con otras instituciones, el protagonismo de eminentes personajes de la vida pública, la organización espacial y temporal interna, entreotros aspectos quegiran en torno a la "institución" como eje y objeto exclusivo del análisis. Estas historias institucionales establecen una división de las transformaciones institucionales por períodos fijos de tiempo, determinados por cambios en los directores o por decisiones administrativas. En síntesis, son historias que hacen visibles los acontecimientos de las instituciones que han sido importantes en la historia de la educación especial en Colombia (o por lo menos en Antioquia).

Una historia social de la educación especial en Colombia es realizada como tesis de maestría en la Universidad Pedagógica Nacional por la educadora especial Maria A melia Gómez (1997). Esta historia cobija el período comprendido entre las décadas de 1900 y 1990. Retoma y fortalece la tesis de José Jaime Díaz (1987) sobreel nacimiento dela educación especial con la creación dela Escuela de Trabajo y Casa de Corrección San Joséen 1914. Para comenzar, incorpora el marco general de análisis histórico sobre lo anormal en Occidente, las explicaciones y prácticas del hombre frente a las anormalidades: infanticidio, abandono, asistencial ismo, beneficencia y mendicidad, entendiendo estas últimas prácticas como un estadio inicial en la historia de la educación especial. Posteriormente plantea la enseñanza como una posi bilidad para la identificación delos niños con debilidades mentales en el marco de la escolarización y el nacimiento de la psicometría, la eugenesia, el aisl amiento y la esterilización como al ternativas para la purificación de la especie humana y la institucional ización de la "deficiencia" como una manera de discriminación. En este punto del análisis, hace un relato cronológico sobre la educación de ciegos y sordos y la aparición de la deficiencia 
mental como un campo de estudio en los países industrializados occidentales. (GÓMEZ, 1997, p.11-60)

Luego analiza los aspectos incidentes en la formación dela educación especial en Colombia iniciando por el asistencialismo como política social par las personas limitadas, la función de los primeros institutos y centros de educación especial, y la relación entre salud y educación como una alternativa para la prevención delas perturbaciones delas personas (GÓMEZ, 1997, p. 61-98) Punto seguido, examina la educación especial en la segunda mitad del siglo XX, mostrando la importancia de la partici pación de los padres y madres a favor de los niños con dificultades, los procesos diferenciados de institucionalización de las poblaciones con limitación auditiva y visual. A simismo, su historia social exponeel lugar dela educación especial dentro de la estructura del MEN con la creación de las aulas remediales y escuel as de retardo mental, los centros de diagnóstico y tratamiento y el centro técnico pedagógico (en el contexto de santa fe de Bogotá). Para terminar, habla sobre los procesos de "integración de discapacitados" en la década de 1990 con su contexto legal y las políticas educativas de apoyo (internacionales y nacionales), la fundamentación conceptual, una experiencia en Bogotá y las estrategias de cambio recientes en el itinerario de la educación especial sobre la base dela política deintegración delos discapacitados (GÓMEZ, 1997, p. 99-172)

De esta manera podemos visual izar varios modos de hacer historia de la educación especial en Colombia, unas maneras espećficas deentender el pasado, el devenir histórico, el valor formativo e investigativo del ejercicio mismo de historiar. Distintos modos que susurran y son como una réplica de otras posibilidades de análisis histórico de la educación y la pedagogía. Son un eco minúsculo que ha sido repetido ante sus oídos.

\section{Una perspectiva CRítica desde la historia de la práctica pedagógica}

Teniendo en cuenta la investigación “El rastro dela diferencia. Historia de la práctica pedagógica de la educación especial en Colombia”, es necesario afirmar que sobre cada uno de estos modos de historiar la educación especial, se pueden establecer al gunas distancias y otros "sentidos deverdad" queresignifiquen las historiassobre y de la educación especial en Colombia, teniendo múltiples efectos directos en su actualidad (por ejemplo, en su estatuto epistemológico, las filiaciones con la pedagogía, la medicina, la psiquiatría y la psicología, sus campos aplicados o sus objetos de saber y de práctica, entre otros; para ampliar ver: YARZA, 2005, 2006b)

En términos generales, se puede aseverar que contrario a sus planteamientos generales, la pedagogía deanormales apropiada einstitucionalizada en Colombia entre 1921 y 1948, se sostuvo -también- en referentes pedagógicos (la pedagogía activa experimental y de anormales Europea y Estadounidense); sus instituciones emergieron como necesidad imperante para disminuir los problemas deenseñanza, como posibilidad deprevenir y proteger el contagio de la población 
"normal", como instituciones profilácticas dela anormalidad y la degeneración, como alternativa educativa para los anormales educables, los retrasados pedagógicos, médicos o morbosos, los díscolos, los débiles mentales; su práctica discursiva y sus conceptos tuvieron un funcionamiento mucho más complejo que lo descrito por unas cuantas líneas someras de recuerdo; se omite la existencia del maestro de anormales, del pedagogo especialista y del médico escolar como sujetos activos y emplazados en esta discursi vidad; oculta las relaciones depoder-saber quemovilizan sus procesos de apropiación e institucionalización, su relación con los saberes modernos, entre otros elementos (para ampliar ver: YARZA, 2005, 2006a, 2006b; YARZA; RODRÍGUEZ, 2004, 2005, 2007).

También podemosindicar los aportes decada uno deellos a un posible programa de investigaciones de pedagogía comparada sobrela educación especial en Colombia eH ispanoamérica. Díaz, Salinas, Duartey Paez permiten identificar algunos "hitos" generales y unas posturas claras. En cuanto a lo primero, es importante la identificación de las fechas de creación de la División Nacional de Educación Especial en 1968, el Programa de Aulas especiales en 1974 o la vinculación al sistema educativo nacional en 1976. Estos "hitos" pueden redimensionarse e interpretarse desde perspectivas de análisis críticoepistemológico que muestren conjuntos de relaciones y cruces de registros que harían emerger hitos locales o singulares con grandes implicaciones para la historia dela educación especial colombiana y latinoamericana.

En cuanto a lo segundo, sepueden formular tres críticas puntuales. En primer lugar, afirmar que la "formación" de maestros de educación especial comienza a finales de 1950 es una imprecisión, puesto que desde la década de 1920 seformaban "maestros deanormales" al interior delas escuelas deciegos, las casas de menores, las escuelas especiales, los servicios médico-escolares o las Juntas Técnicas. En segundo lugar, toman como punto de partida el desarrollo dela historia de la educación especial en Europa homologando y determinando los períodos históricos en su misma secuencia, estableciendo una relación determinista infranqueablee indisoluble desdesus enfoques deanálisis. En tercer lugar, plantean queel período inicial einaugurador dela educación especial es institucionalizador y segregador, desconociendo ("en parte") las justificaciones, explicaciones e intereses construidos en esa época que propiciaron su apropiación, las cuales valoraban la necesidad deinstruir y educar a los denominados "niños anormales" para disminuir las cargas al Estado y la familia, para prevenir a los "estudiantes normales" y, sobretodo, como decía Don Tomás Cadavid y Don David Velásquez (1921, 23), director y médico dela Casa de Menores y Escuela de Trabajo San José, a los anormales era necesario que "[...] se les adiestre para la lucha de la vida, enseñándoles un arte o profesión que los haga independientes por medios honrados."

Por su parte, José Jaime Díaz Osorio permite establecer una mirada cronológica lineal precisa sobrela legislación concernientea la educación especial. Funciona como el texto deBohorquez Casal las (1956) para la historia dela educación 
y la pedagogía en Colombia, es decir, como una obra que delimita una sucesión lineal de hechos, fechas y datos que pueden ser analizados críticamente. Sin embargo, es necesario desmontar su visión teleológica, progresista, evolutiva y humanizadora de la historia de la educación especial en nuestra región. Mirada ingenua, reduccionista y simple que ha tenido unos efectos un tanto "perversos" sobre los imaginarios del pasado, ese pasado instalado en la lejanía de lo que aconteció y no volverá.

Lainvestigación deA ntonio Marín esesencial para conocer una historia dela institución sobrela cual secierneel mito fundacional dela educación especial en el país: la casa demenoresy escuela detrabajo San José. También permiteconocer la existencia de laR evista Estudio y Trabajo (un potentemecanismo deapropiación deestesaber aún sin analizar), los reglamentos, las relaciones con otras instituciones (las Colonias vacacionales, la escuela de díscolos Tomás Cadavid Restrepo, la escuela especial Rafael Uribe Uribe) y las clasificaciones que orientaban la organización y distribución del espacio institucional. Esta información es vital para establecer una lectura distinta sobrelas "fuentes primarias" indicadas por el Archivo $y$, al mismo tiempo, para conocer una linealidad que podría escandirse, zurcirse en una "historia discontinua" desdela pedagogía y las relaciones desaber-poder. Sin embargo, no deja deser una mi rada centrada en una institución, un punto en una red mucho más densa y extendida en la sociedad con objetivos políticos explícitos, reales y transversales a lo social. En esta misma dirección es importante la tesis del historiador Hugo de Jesús Pérez sobrela escuela deciegos y sordomudos.

Por su parte, Amelia Gómez posibilita percibir al gunas filiaciones con las transformaciones sociales y económicas del siglo XX, aspecto importante al momento deconstruir una "pequeña arqueología" delos procesos de apropiación de la pedagogía de anormales y la educación especial en Colombia. Sus apuntes son vital es para las enunciaciones pedagógicas desdeun punto de vista que no es exclusivamente "social". A demás, porqueavanza en los hechos y acontecimientos sociales de la segunda mitad del siglo pasado (período que todavía merece una exploración sistemática desde una mi rada histórico-pedagógica que demarquelas diferencias y continuidades entrela pedagogía de an ormal es y la institucionalización nacional de laeducación especial).

Estos modos dehacer historia merecen ser objeto deotras críticas puntuales que pueden constituirse en la antesala obligatoria para marcar al gunas huellas y senderos de un redireccionamiento crítico en los análisis históricos sobre la educación especial en Colombia (con potencial de hacerlo también para Hispanoamérica). En términos generales y provisionales las críticas son las siguientes:

1. U n efecto depoder mediantela construcción deLA imagen del "pasado" en la educación especial. El primer análisis realizado desdeuna “historia dedatos" por JoséjaimeDíaz Osorio en 1987, se constituyó en el referente obligatorio para todos los demás estudios históricos quelesucedieron. Todos sin excepción lo mencionan 
o lo retoman. La mirada de Díaz instauró una imagen del "pasado" quieto, lineal, ascendente, anquil osado en la Casa de Menores San Joséy la Escuela de Ciegos y Sordomudos en Antioquia en tanto puntos incuestionables del nacimiento dela educación especial en Colombia. El pasado como lo acontecido sin reservas ni remedios, sin posibilidades de cambios, una especie de pasado ensimismado, una visión única, global y unificada sobrela historia dela educación especial.

2. La mayoría de los estudios sesitúan en los "imaginarios" actuales sobreel pasado para analizar y describir la historia de la edu cación especial. Las historias de datos, institucionales y sociales, realizadas por historiadores, administradores, profesores universitarios o maestros, se han instalado en una simplificación por binomiosu oposición de prácticas representacionales en la educación especial (Cfr. SKLIAR, 1998, 2003, 2005; YARZA , 2006b): asistencial y pedagógico, segregación e integración, institucionalización y desinstitucionalización o exclusión einclusión como un modus operan di para diferenciar etapas o períodos históricos (además del uso de metáforas biológico-evolutivas de crecimiento). Se sitúan en una intencionalidad del presente para remirar el pasado con terminologías y descripciones total mente permeadas por el imaginario de los "binomios opuestos".

3. Por tanto, la mayoría deestos modos dehacer historia están cimentados en una per spectiva un tanto "ingenua einocente". En todos sevalora y rescata una supuesta evolución hacia mejores formas deatención educativa para los "anormales", una humanización ascendente e innegable. Esta apreciación sobre un humanismo rimbombante carece de toda postura crítica y reflexiva, dejando de instalar en el escenario preguntas epistémicas, dedominios desaber, derelaciones de producción simbólica y económica, de las microfísicas de poder, etc. En esta medida, no sólo los trabajos históricos sobre la educación especial, sino la educación especial en sí misma requiere con urgencia de investigaciones preferiblemente interpretativas, cualitativas y críticas, dereflexiones teóricas o deensayos que posi biliten desacoplar la hegemonía subrepticia de una "[...] estructuración moderna, experimental y utilitarista [que] impregna los zócal os de saber que rigen la "educación especial" en el presente[...]" (YARZA; RODRÍGUEZ, 2005, p. 67).

4. Como consecuencia inmediata, son portadores de una "apolitización implícita" del análisis histórico. En la medida que no sospechan sobre el supuesto progreso continuo, la inevitable y evidente mejoría, el indiscutible ascenso de un "cuidado más humano y pedagógico", etc.; están imposibilitados para reconocer su postura política en el presente, es decir, concienciarse del compromiso políticopedagógico anclado en un "presente" cambiante y contradictorio (como señala Freire). Al mismo tiempo, no permite visualizar la importancia radical del conocimiento y el reconocerse en la historia con su potencial transformativo de subjetividades e institucional idades históricamente situadas y fechadas. ${ }^{6}$

\footnotetext{
${ }^{6}$ En esta dirección, es importante mencionar y remitir a los trabajos realizados por Kim Reid y Jan Weatherly Valle (2004) sobre “la práctica discursiva delas dificultades deaprendizaje” y por Curt Dudley-Marling (2004) sobre "la construcción social de las dificultades de aprendizaje" en el contexto delas Teorías Curriculares y de análisis del discurso pedagógico real izado en los Estados Unidos.
} 
5. Por último, y no menos importante, enceguecela au sencia de "pedagogía" en los análisis históricos (con excepción de A ntonio Marín, aunque lo haga desde una correspondencia externa y no como un campo de análisis). Tal vez sea un tanto radical en la apreciación, pero lo que se quiere hacer visible es quela forma en queopera la "pedagogía" en los análisis históricos sobre la educación especial es mínima, inconsciente, acrítica einstrumental; siendo necesario profundizar en los potenciales existentes en una pedagogía histórica en constanteretroalimentación entre la pedagogía como saber y la historia como escenario para tomar como objeto deanálisis lahistoricidad dela educación especial.

A hora bien, no podemos terminar con este escrito sin bosquejar un mínimo deplanteamientos quecatapulten las historias sobrey dela educación especial desde perspectivas histórico-pedagógi cas críticas y epistémicas. Por consiguiente, a continuación se esbozan al gunas pistas para navegar hacia unos modos de historiar desde un puerto diferentea lo acostumbrado.

a. Es importanteasirse una óptica, a una caja de herramientas quenos permita deshacer o de-construir el estado actual dela imagen sobre "el pasado" dela educación especial. En esto consistiría continuar con el rescate dela práctica pedagógica dela educación especial desdeuna “tradición" concreta deanálisis (ZULUAGA , 2005). Mirar con la microfísica del poder, con la problematización de los dominios de saber, de los procesos de subjetivación, de la constitución histórica de la verdad y de las técnicas, de la configuración de los regímenes institucionales, la movilidad delos conceptos y las conceptual izaciones, entre otros componentes significativos que nos permitan la resignificación histórica del presente.

b. Es necesario el acercamiento a métodos históricos diversos que hagan visibles diferentes acontecimientos, relaciones culturales, sociales, políticas, etc.; por ejemplo, continuar con la cercanía no solamentehacia las analíticas de Michel Foucault, sino también con Michel DeCerteau (historias dela cotidianidad) o con Phillipe Joutard (historias orales y devida). Asegurando el incremento de estas maquinarias analíticas y metódicas se podría afianzar un programa de investigaciones sobre historias de la educación especial desde su articulación con el Campo Conceptual dela Pedagogía (ECHEVERRI, 2001, 2002, 2004), lo cual implica, al mismo tiempo, amplificar las relaciones con la "pedagogía" misma (pedagogía crítica, pedagogía diferenciada, etc.)

c. También es necesario pensar la articulación efectiva deestas nuevas historias de la educación especial con los planes de formación inicial de maestros y con la formación permanente de maestros en ejercicio. La historia no es para dejarla en anaqueles y estanterías, ni mucho menos en revistas de poca circulación o en congresos de especial istas. Nuestros estudios sobrehistoria de la pedagogía y la educación (dentro de los cuales estarían las historias sobrey dela educación especial) deben tener mínimamente efectos en la formación del magisterio y en la solidificación de una comunidad académica; porque, sin duda al guna, su 
mayor utilidad consistiría en posibilitarnos pensar más allá denuestros límites mediante una crítica radical hacia la normalidad, no ala "anormalidad" (cfr. Skliar, 2005)

No obstante, es urgente pensar de otro modo instalados en la historia y en la pedagogía, implicando queno debamos dejar decincel ar bloques de piedra 0 mármol para trazar unas huellas, al gunos rastros para que cual quiera los lea... El olvido deberá combatirse con el recuerdo, una memoria que mantenga vivas las preguntas y las problematizaciones, las imágenes, las presencias y ausencias del otro en nosotros. Un interrogar desde nuestra actualidad que rescata el valor altamente pedagógico de rememorar el pasado desde lo acontecido, desde el devenir, la transformación y los cambios insospechados, tal vez, por que precisamente están olvidados. La intencionalidad es educar la memoria, "[...] la capacidad de recordar activamente el pasado, y la capacidad de imaginarnos a nosotros mismos evocando experiencias pasadas [que] nos permita dar sentido, no solamente a lo que ocurrió, sino también a nuestro presente como algo relacionado con un pasado quetodavía puede enseñarnos al go." (Bárcena, 2001, 101) Así pues, tenemos un imperativo formativo con el presente: ponerleun alto al incremento y expansión de la des-memorización de la cultura, la historia, la pedagogía y, para nuestro caso, dela educación especial.

\section{REFERÊNCIAS}

BARCENA ORBE, F. La esfinge muda: el aprendizaje del dolor después de Auschwitz. Barcelona: A nthropos, 2001.

. El delirio de las palabras: ensayo para una poética del comienzo. España: Herder, 2004.

BOHÓRQUEZ CASALLAS, L. A. La evolución educativa en Colombia. Bogotá: Cultural Colombiana, 1956.

CACUA PRADA, A. H istoria dela educación en Colombia. Bogotá: A cademia Colombiana de Historia, 1997.

CASTRO, J. O. Historia dela educación y la pedagogía. Una mirada a la configuración de un campo de saber, In: HENAO WILLES, M. y CASTRO, J. O. (Comp.) Estados del arte de la investigación en Educación y Pedagogía en Colombia I. Bogotá: ICFES, Colciencias, Sociedad Colombiana dePedagogía, 2000. p. 235-278.

DÍAZ OSORIO, J. J. La educación especial y sus acciones en el departamento de Antioquia. Medellín: Secretaría de Educación y Cultura, 1987.

DUDLEY-MARLING, C. The Social Construction of Learning Disabilities. Journal of Learning D isabilities, Texas (Estados Unidos), v. 37, n. 6, p.482-489, 2004.

ECHEVERRI SÁ NCHEZ, J. A. Premisas conceptuales del Dispositivo Formativo Comprensivo. Revista Educación y Pedagogía, Medellín, v. 8, n. 16, p. 71-105, 1996.

. Postdata de una investigación concluida. (ACIFORMA) Cuadernos Pedagógicos, Medellín, v. 15, p. 35- 42, 2001.

. Reflexiones acerca de la formación de maestros y la investigación. In: NOGUERA, C. E., et al. Investigación pedagógica en Colombia. Medellín: Maestros Gestores deN uevos Caminos, Corporación REGIÓN, 2002. p. 202-223.

Cartas a Clotilde. Revista Colombiana de Educación, Bogotá, n. 47, p. 254-289, 2004. 
FACULTAD DE EDUCACIÓN, UNIVERSIDAD DE ANTIOQUIA, MINISTERIO DE EDUCACIÓN NACIONAL. I Foro Nacional. Pertinencia social y educativa de la formación de maestros y maestras en educación especial. 12 y 13 de Octubre. Sede de Investigación Universitaria. Medellín: Facultad de Educación, CD Rom, 2006.

FAULKNER, W. A bsolón, A bsolón. Argentina: Emece, 1951.

GARCíA, J. C. Historia de la instrucción pública en Antioquia. Medellín: Editorial Universidad de Antioquia, 1962.

GÓMEZ, M. A. A puntes para una historia de la educación especial en Colombia. 1997. 187f. Tese(Maestría en Historia dela educación) - Universidad Pedagógica N acional, Bogotá.

HELG, A. La educación en Colombia: 1918-1957 y La educación en Colombia: 1958-1980. In: La nueva H istoria de Colombia. Bogotá: Planeta, 1987. V. 4

HERRERA, M. M odernización y Escuela N ueva en Colombia. 1914-1951. Bogotá: Plaza \& Janes, 1999.

JARAMILLO URIBE, J. H istoria dela pedagogía como historia de la cultura. Bogota: Universi dad Nacional deColombia, 1978.

. El proceso de la educación en la República (1830-1886). In: N ueva Historia de Colombia. Era republicana. Bogotá: Planeta, 1984. p. 223-250, v. 2.

. La educación durante los gobiernos liberales. 1930-1946. In: N ueva Historia de Colombia. Educación y ciencias, luchas de la mujer, vida diaria. Bogotá: Planeta, 1989. p. 87-110, v. 4.

LE BOT, I. Educación e ideología en Colombia. Medellín: La Carreta, 1979.

MARÍN CASTAÑO, J. A. R econstrucción histórica de la escuela de trabajo San José 1914-1991. Medellín: Fundación Universitaria Luis Amigo, 1992.

MARTÍNEZ BOOM, A. y NARADOWSKI, M. (Comp) Escuela, H istoria y Poder. (M iradas desdeA mérica Latina). Argentina: Novedades Educativas, 1996.

PAEZ ESCOBAR, M. A proximación histórica de la educación especial en Colombia. V enciendo Barreras, Bogotá, v. 7, n. 1, p. 9-10, 1994.

PEDRAZA, Z. En Cuerpo y A Ima. V isiones del Progreso y de la Felicidad. Bogota: Universidad de los Andes, 1999.

PÉREZ, H. de J. Historia de la Educación para limitados visuales y auditivos en A ntioquia, 1925-2002: el caso del colegio de atención al limitado visual y auditivo francisco luis hernández betancur. 2002. 211f. Tese (Historia) - Departamento deH istoria, Universidad deA ntioquia, Medellín.

QUICENO CASTRILLÓN, H. Crónicas históricas de la Educación en Colombia. Bogotá: Cooperativa Editorial Magisterio, Grupo Historia dela Práctica Pedagógica, 2003.

REID, D. K.; WEATHERLY VALLE, J. The discursve practice of learning disability: implications for instruction and parent-school relations. Journal of Learning D isabilities, Texas, v. 37, n. 6, p. 466-481, 2004. ROSELLI, H. H istoria de la psiquiatra en Colombia. Colombia: Horizonte, 1968.

SAENZ OBREGON, J.; SALDARRIAGA, O.; OSPINA, A. M irar la Infancia: pedagogía, moral y modernidad en Colombia. 1903 - 1946. Bogotá: Foro Nacional por Colombia, Universidad de Antioquia, Colciencias, 1997. v. 1 e2.

SALDARRIAGA VÉLEZ, O. Del oficio de maestro. Prácticas y teorías de la pedagogía moderna en Colombia. Bogotá: Cooperativa Editorial Magisterio, Grupo Historia dela Práctica Pedagógica, 2003.

SA LINAS, L. Evolución histórica de la Educación Especial en Colombia. In: U n siglo de educación en Colombia. Bogotá: Ministerio de Educación Nacional, Oficina de Planeación del Sector Educativo, 1988. p. 47-49.

SILVA OLARTE, R. La educación en Colombia. 1880-1930. In: N ueva H istoria de Colombia. Educación y ciencias, luchas de la mujer, vida diaria. Bogotá: Planeta, 1989. p. 61-86, v. 4.

SKLIAR, C. Repensando la Educación Especial. R evista A Iternativas, San Luis, v. 3, n. 13, p. 19-36, 1998. - ¿Y si el otro no estuviera ahí? N otas para una pedagogía (improbable) dela diferencia Buenos Aires: Miño y Dávila Editores, 2003. 
SKLIAR, C. Poner en tela dejuicio la normalidad, no la anormalidad. Políticasy falta de políticasen relación con las diferencias en educación. Revista Educación y Pedagogía, Medellín, v. 27, n. 41, p. 922, 2005.

SOCIEDAD COLOMBIANA DE HISTORIADORES. M emorias del XIII Congreso Col ombiano deH istoria, 13, 2006. A nais... Bucaramanga: UIS, UNAL, CDRom, 2006.

YARZA DE LOSRÍOS, A. Travesías: notas para una pedagogía y una epistemología dela educación especial en Colombia. Revista de Pedagogía, Caracas, v. 26, n. 76, p. 281-306, 2005.

. Algunos modos de historiar la educación especial en colombia: una mirada crítica desdela historia de la práctica pedagógica. In: SOCIEDAD COLOMBIANA DE HISTORIADORES, M emorias XIII Congreso Colombiano de Historia13, 2006. A nais... Bucaramanga: UIS, UNAL, 2006 a.

"Pedagogía de anormales" y formación de maestros: ¿una mirada desde el déficit? 19211948. In: CONGRESO INTERNA CIONAL DE PEDA GOGÍA: Diversidad educativa + inclusión, 1 , 2006. A nais..... Medellín: Comunicando Sentidos, FUN DIVE, CD-Rom, 2006b.

YARZA DE LOSRÍOS, A.; RODRÍGUEZ RAVE, L. M. Pedagogía, idiotas y anormales: una historia dela pedagogía de anormal es en Colombia, 1870-1930. Revista A Iternativas, Serie Espacio Educativo, San Luis, v. 9, n. 35-36, p. 115-130, 2004.

.H orizonteconceptual y tecnologías médico-psico-pedagógicasen la “pedagogía deanormales" en Colombia: 1920-1940. Revista Educación y Pedagogía, Medellín, v. 27, n. 41, p. 55-68, 2005.

Educación de anormales, saber pedagógico y modernidad a principios del siglo XX en Colombia. In: Revista Historia y Sociedad, Medellín. En prensa, 2007.

ZULUAGA GARCÉS, O. L. Pedagogía e H istoria. La historicidad de la Pedagogía. La enseñanza, un objeto de saber. Bogotá: Universidad deA ntioquia, Anthropos, 1999.

. Foucault: una lectura desde la práctica pedagógica. In: ZULUAGA, O. L., et al. Foucault, la Pedagogía y la Educación. Pensar de otro modo. Bogotá: Cooperativa Editorial Magisterio, Grupo Historia de la Práctica Pedagógica, UPN, IDEP. 2005. p. 11-37.

ZULUAGA, O. L., et al. Pedagogía y epistemología. Bogotá: Cooperativa Magisterio, Grupo Historia de la Práctica Pedagógica, 2003.

Recebido em 17/ 05/ 2007

A provado em 30/ 08/ 2007 